\title{
Zelluläre Immuntherapien als Kombinationspartner für die Immun-Checkpoint-Blockade
}

\author{
Claudia Rössig \\ Pädiatrische Hämatologie und Onkologie, Universitätsklinikum Münster, Deutschland
}

Neben der Checkpoint-Inhibition gelten zelluläre Immuntherapien als vielversprechende Ergänzungsoption zu den etablierten Behandlungskonzepten [1]. Die allogene hämatopoetische Stammzelltransplantation (HSZT) hat das therapeutische Potenzial der zellulären Immuntherapie bei chemotherapierefraktären malignen hämatologischen Erkrankungen verdeutlicht. So können Graft-versus-Leukämie (GvL)-Effekte, gezielt verstärkt durch Transfusionen von Spenderlymphozyten, maßgeblich zu anhaltenden Remissionen beitragen [2]. Allerdings treten durch die Alloreaktivität der SpenderT-Zellen häufig unerwünschte Wirkungen im Sinne von Graftversus-Host-Erkrankungen auf.

Mit dem Ziel, spezifischere T-Zell-Therapien für die Behandlung von Krebs zu entwickeln, wurden experimentelle Verfahren eingesetzt, mit denen tumorreaktive autologe T-Zellen in vitro expandiert werden können. In der Tat gelang es z.B. durch adoptiven Transfer autologer Epstein-Barr-Virus (EBV)-spezifischer T-Zellen, vollständige Remissionen bei Patienten mit refraktären EBV-assoziierten Lymphomen zu induzieren [3]. Allerdings exprimieren nur sehr wenige Krebserkrankungen virale Antigene, und die Expansion autologer T-Zellen gegen nicht virale tumorassoziierte T-Zellen hat sich aus deutlich schwieriger erwiesen. Viele dieser Antigene sind in geringer Dichte auch auf gesunden Zellen exprimiert, sodass aufgrund von Mechanismen der Immuntoleranz nur sehr wenige antigenspezifische T-Zellen im Repertoire des Patienten verbleiben. Auch weisen Tumorzellen erhebliche Defizite der Antigenpräsentation auf, die die Erkennung durch spezifische T-Zellen verhindern.

\section{Reprogrammierung von T-Zellen gegen tumorassoziierte Antigene}

Das genetische Engineering von T-Zellen erlaubt heute die Generierung größerer Mengen therapeutisch nutzbarer
T-Zellen. Mit dieser Methode können zytotoxische T-Zellen mit chimären T-Zell-Rezeptoren (chimeric antigen receptors, CARs) ausgestattet werden, die tumorassoziierte Oberflächenantigene erkennen. In CARs ist die Antigen-Erkennungsdomäne eines Antikörpers mit der signaltransduzierenden $\zeta$-Untereinheit des T-Zell-Rezeptors verbunden [4]. Da Tumorzellen häufig nicht über ausreichende Mengen kostimulatorischer Liganden verfügen, die für die vollständige Aktivierung von T-Zellen benötigt werden, werden kostimulatorische Signalkomponenten in die CARs integriert [5, 6]. Die Interaktion der Rezeptoren mit dem entsprechenden Antigen führt zu einer Signalweiterleitung und antigenspezifischen Aktivierung der T-Zelle. Da die Antigenspezifität der genetisch modifizierten T-Zellen durch die Erkennungsdomäne eines monoklonalen Antikörpers definiert wird, erfolgt die Interaktion mit der Tumorzelle mit Oberflächenantigenen unabhängig von der Antigenpräsentation, sodass Immunresistenzmechanismen umgangen werden.

Die meisten der bisherigen klinischen Studien CARsbasierter Zelltherapie wurden mit dem B-Zell-Antigen CD19 bei chronischen lymphatischen Leukämien (CLL) oder akuten lymphoblastischen Leukämien (ALL) vom BVorläufer-Typ durchgeführt [6, 7]. Da CD19 nicht nur auf leukämischen Blasten exprimiert wird, sondern auch auf weiteren Zellen der B-Zell-Reihe, kommt es infolge der CD19-spezifischen CARs-Therapie zu einem transienten oder auch anhaltenden B-Zell-Defekt mit Hypogammaglobulinämie, der eine Substitution mit Immunglobulinen erforderlich machen kann. Aktuelle frühe klinische Daten stimmen vorsichtig optimistisch, dass die Therapie mit CARsgenmodifizierten T-Zellen einen wesentlichen Beitrag zur Therapie von B-Zell-Malignomen und möglicherweise weiteren Krebserkrankungen leisten kann. Sowohl bei CLL als auch bei CD19-positiver ALL konnten durch CARs-basierte Zelltherapie bei einigen therapierefraktären Patienten selbst nach vorausgegangenen allgenen Stammzelltransplantatio-

\begin{tabular}{ll}
\hline KARGER & (2) 2014 S. Karger GmbH, Freiburg \\
2296-5270/14/3717-0014\$39.50/0 \\
$\begin{array}{l}\text { Fax+49 7614520714 } \\
\text { Information@Karger.com } \\
\text { www.karger.com }\end{array}$ & $\begin{array}{l}\text { Accessible online at: } \\
\text { www.karger.com/ort }\end{array}$
\end{tabular}


nen anhaltende Remissionen erreicht werden. Diese korrelierten mit einer Persistenz CARs-modifizierter T-Zellen im Blut der Patienten [6, 7].

Insbesondere für den Einsatz von CARs-modifizierten T-Zellen bei soliden Tumoren wird ein erweitertes Verständnis immunsuppressiver Interaktionen im Mikromilieu des Tumors notwendig sein, die das Homing und die Funktion der therapeutischen T-Zellen beeinträchtigen. Möglicherweise können kombinierte Ansätze mit Immun-Checkpoint-Inhibi- toren dazu beitragen, zellbasierte Immuntherapien sinnvoll in ein optimiertes immunologisches Behandlungskonzept $\mathrm{zu}$ integrieren.

\section{Disclosure Statement}

Die Autorin war Referentin beim «4. Interdisziplinären Expertenforum», das von Bristol-Myers Squibb unterstützt wurde.

\section{Literatur}

1 Couzin-Frankel J: Breakthrough of the year 2013. Cancer immunotherapy. Science 2013;342:14321433.

2 Kolb HJ, Schattenberg A, Goldman JM, et al.: Graft-versus-leukemia effect of donor lymphocyte transfusions in marrow grafted patients. Blood 1995;86:2041-2050.

3 Bollard CM, Gottschalk S, Torrano V, et al.: Sustained complete responses in patients with lymphoma receiving autologous cytotoxic $\mathrm{T}$ lymphocytes targeting Epstein-Barr virus latent membrane proteins. J Clin Oncol 2014;32:798-808.
Roessig C, Bollard CM, Nuchtern JG, et al.: Targeting of G(D2)-positive tumor cells by human T lymphocytes engineered to express chimeric T-cell receptor genes. Int J Cancer 2001;94:228-236.

5 Song DG, Ye Q, Carpenito C, et al.: In vivo persistence, tumor localization, and antitumor activity of CAR-engineered $\mathrm{T}$ cells is enhanced by costimulatory signaling through CD137 (4-1BB). Cancer Res 2011;71:4617-4627.

6 Porter DL, Levine BL, Kalos M, et al.: Chimeric antigen receptor-modified $\mathrm{T}$ cells in chronic lymphoid leukemia. N Engl J Med 2011;365:725-733.
7 Brentjens RJ, Davila ML, Riviere I, et al.: CD19targeted $\mathrm{T}$ cells rapidly induce molecular remissions in adults with chemotherapy-refractory acute lymphoblastic leukemia. Sci.Transl.Med 2013;5: $177 \mathrm{ra38.}$

7 Kalos M, Levine BL, Porter DL, et al.: T cells with chimeric antigen receptors have potent antitumor effects and can establish memory in patients with advanced leukemia. Sci Transl Med 2011;3:95ra73. 